FACTA UNIVERSITATIS (NIŠ)

Ser. Math. Inform. Vol. 36, No 3 (2021), 627 - 641

https://doi.org/10.22190/FUMI210227047C

Original Scientific Paper

\title{
AN EXAMINATION OF THE CONDITION UNDER WHICH A CONCHOIDAL SURFACE IS A BONNET SURFACE IN THE EUCLIDEAN 3-SPACE
}

\author{
Muradiye Çimdiker Aslan ${ }^{1}$, Gülşah Aydın Şekerci ${ }^{2}$ \\ 1 Muradiye Çimdiker Aslan, Kırklareli University, Department of Mathematics, \\ 39100 Kirklareli, Turkey \\ ${ }^{2}$ Süleyman Demirel University, Department of Mathematics, \\ 32260 Isparta, Turkey
}

\begin{abstract}
In this study, we examine the condition of the conchoidal surface to be a Bonnet surface in Euclidean 3-space. Especially, we consider the Bonnet conchoidal surfaces which admit an infinite number of isometries. In addition, we study the necessary conditions which have to be fulfilled by the surface of revolution with the rotating curve $c(t)$ and its conchoid curve $c_{d}(t)$ to be the Bonnet surface in Euclidean 3-space.
\end{abstract}

Keywords. Conchoidal surface, Bonnet surface, Euclidean 3-space.

\section{Introduction}

The conchoid of Nicomedes, which is called by the Greek geometer Nicomedes's name, was originally contrived around $200 \mathrm{BC}$ to trisect an angle and duplicate the cube. For any curve and a fixed point, let a straight line, which meets the curve at the point $Q$, is drawn through the fixed point. If $P$ and $R$ are points on this line such that $R Q=Q P=$ const., then the conchoid of curve with respect to the fixed point is the locus of $P$ and $R$ [12].

The conchoids play an important role in many applications as the construction of buildings, astronomy [9], optics [2], physics [19]. Although the

Received Febryary 27, 2021. accepted July 242021.

Communicated by Uday Chand De

Corresponding Author: Gülşah Aydın Şekerci, Süleyman Demirel University, Department of Mathematics, 32260 Isparta, Turkey, gulsahaydin@sdu.edu.tr

2010 Mathematics Subject Classification. Primary 53A05

(C) 2021 by University of Niš, Serbia | Creative Commons License: CC BY-NC-ND 
conchoidal constructions were extensively mentioned by the ancient Greeks in the seventeenth century, they have been recently addressed by different authors, too. One of these has been put forward by Odehnal. He obtained a generalized conchoid transformation considering a construction with the help of cross ratios [13]. Moreover, Peternel, etc. presented the conchoidal surface of rational ruled surfaces, the conchoidal surfaces of spheres, the conchoids and the pedal surfaces [15, 16, 17].

Surfaces, which admit a one-parameter family of isometries preserving the mean curvature, have been proposed by Bonnet and although Bonnet raised these surfaces [3], the term "Bonnet surface" was firstly used by Lalan [11]. Bonnet showed that all surfaces with the constant mean curvature can be isometrically mapped to each other and the deformable surfaces with the non-constant mean curvature are the isothermic Weingarten surfaces which can be deformable to the revolution surfaces. After that, many mathematicians have contributed these surfaces $[18,10,7,1]$.

Bonnet surfaces may be broken up into three types which is described as follows:

(i) Surfaces of the constant mean curvature other than the plane or the sphere.

(ii) Isothermic Weingarten surfaces of the non-constant mean curvature which admit a one parameter family of geometrically distinct non-trivial isometries.

(iii) Surfaces of the non-constant mean curvature that admit a single non-trivial isometry [10].

In [4], the authors studied the conchoidal surfaces, the surfaces of revolution given with the conchoid curve and their geometrical properties in Euclidean 3space. In our work, using the geometric properties obtained for conchoidal surfaces in reference [4], we have examined the conditions under which the conchoidal surface and the surface of revolution given with conchoid curve is a Bonnet surface in Euclidean 3- space. According to that, we get the following results:

(1) If a regular surface $M$ and a conchoidal surface $M_{d}$ are minimal, then they are the surfaces of the type $(i)$ which can be recognised by an infinite number of isometries preserving the principal curvatures.

(2) The surfaces $M$ with the radius function $r\left(u_{0}, v\right)$ or $r\left(u, v_{0}\right)$ are the surfaces of the type (ii) which admit an infinite number of isometries. Also, the result is similar for the conchoidal surfaces $M_{d}$.

(3) If a regular surface $M$ and a conchoidal surface $M_{d}$, which are the surfaces of revolution generated by the rotating curve and its conchoid curve, are minimal, then they are the surfaces of the type $(i)$ which can be recognised by an infinite number of isometries preserving the principal curvatures.

(4) If a regular surface $M$ and a conchoidal surface $M_{d}$, which are the surfaces of revolution generated by the rotating curve and its conchoid curve with the radius function $r\left(u_{0}, v\right)$ or $r\left(u, v_{0}\right)$, are the surfaces of the type $(i i)$ which admit an infinite number of isometries.

\section{Preliminaries}

Let $M$ be a smooth surface in $\mathbb{E}^{3}$ given with the patch $X(u, v)$ for 
$(u, v) \in D \subset E^{3}$. The tangent space to $M$ at an arbitrary point $p$ of $M$ is spanned by $\left\{X_{u}, X_{v}\right\}$. Let $N$ be the unit normal vector field of the surface $M$ defined by $N=\frac{X_{u} \times X_{v}}{\left\|X_{u} \times X_{v}\right\|}$. The first fundamental form $I$ and the second fundamental form II of the surface $M$ are

$$
I=e d u^{2}+2 f d u d v+g d v^{2}, \quad I I=l d u^{2}+2 m d u d v+n d v^{2},
$$

respectively, where

$$
e=\left\langle X_{u}, X_{u}\right\rangle, f=\left\langle X_{u}, X_{v}\right\rangle, g=\left\langle X_{v}, X_{v}\right\rangle,
$$

and

$$
l=\left\langle X_{u u}, N\right\rangle, m=\left\langle X_{u v}, N\right\rangle, n=\left\langle X_{v v}, N\right\rangle .
$$

In [8], the Gaussian curvature $K$ and the mean curvature $H$ are

$$
K=\frac{l n-m^{2}}{e g-f^{2}}, \quad H=\frac{e n-2 f m+g l}{2\left(e g-f^{2}\right)} .
$$

A surface $M$ in $\mathbb{E}^{3}$ is called Weingarten surface if there exists a non-trivial functional relation

$$
\Omega(K, H)=0
$$

with respect to its Gaussian curvature $K$ and its mean curvature $H$, where $\Omega$ is the Jakobian determinant [14].

If a surface $M$ in $\mathbb{E}^{3}$ has the coefficients of first fundamental form which satisfy the conditions $e=g, f=0$, then it is called isothermic [5]. According to [18], the isothermic surface provides the condition

$$
\frac{\partial^{2}}{\partial u \partial v}\left(\log \frac{g}{e}\right)=0 .
$$

We assume a smooth surface $M \subset E^{3}$ and a fixed reference point $O$ which can be considered as the origin of a cartesian coordinate system. Let $M$ is described by a polar representation

$$
X(u, v)=r(u, v) s(u, v)
$$

with $\|s(u, v)\|=1$. Considering $s(u, v)=(\cos u \cos v, \sin u \cos v, \sin v)$ of the unit sphere $S^{2}$, so $s(u, v)$ and $r(u, v)$ are called spherical part and radius function of $X(u, v)$, respectively.

In $[17,15]$, the one-sided conchoidal surface $M_{d}$ of $M$ is derived by adding $d \in \mathbb{R}$ to the radius function $r(u, v)$ and thus $M_{d}$ admits the polar representation

$$
M_{d}(u, v)=(r(u, v)+d) s(u, v) .
$$


Let $M$ be a regular surface given with the parametrization (2.7). Then the coefficients of the first fundamental form of the surface $M$ are

$$
\begin{aligned}
e & =r^{2} \cos ^{2} v+r_{u}^{2}, \\
f & =r_{u} r_{v}, \\
g & =r^{2}+r_{v}^{2} .
\end{aligned}
$$

Additionally, its Gaussian curvature and its mean curvature are

$$
\begin{aligned}
K= & -\frac{1}{r^{2} A^{2}}\left[r r_{u v} \cos v-2 r_{u} r_{v} \cos v+r r_{u} \sin v\right)^{2} \\
& \left.-\cos ^{2} v\left(2 r_{u}^{2}+r r_{v} \sin v \cos v+r^{2} \cos ^{2} v-r r_{u u}\right)\left(2 r_{v}^{2}+r^{2}-r r_{v v}\right)\right],
\end{aligned}
$$

and

$$
\begin{aligned}
H= & -\frac{1}{2 r^{2} A^{3 / 2}}\left[\cos v\left(2 r_{u}^{2}+r r_{v} \sin v \cos v+r^{2} \cos ^{2} v-r r_{u u}\right)\left(r^{2}+r_{v}^{2}\right)\right. \\
& +\cos v\left(2 r_{v}^{2}+r^{2}-r r_{v v}\right)\left(r^{2} \cos ^{2} v+r_{u}^{2}\right) \\
& \left.+2 r_{u} r_{v}\left(r r_{u v} \cos v-2 r_{u} r_{v} \cos v+r r_{u} \sin v\right)\right],
\end{aligned}
$$

where $A=\left(r^{2}+r_{v}^{2}\right) \cos ^{2} v+r_{u}^{2}$. Also, if $M_{d}$ is a conchoidal surface given with the parametrization (2.8), its Gaussian curvature and its mean curvature are

$$
\begin{aligned}
\widetilde{K}= & -\frac{1}{(r \pm d)^{2} A^{2}}\left[\left((r \pm d) r_{u v} \cos v-2 r_{u} r_{v} \cos v+(r \pm d) r_{u} \sin v\right)^{2}\right. \\
& -\cos ^{2} v\left(2 r_{u}^{2}+(r \pm d) r_{v} \sin v \cos v\right. \\
& \left.\left.+(r \pm d)^{2} \cos ^{2} v-(r \pm d) r_{u u}\right)\left(2 r_{v}^{2}+(r \pm d)^{2}-(r \pm d) r_{v v}\right)\right],
\end{aligned}
$$

and

$$
\begin{aligned}
\widetilde{H}= & -\frac{1}{2(r \pm d)^{2} A^{3 / 2}}\left[\operatorname { c o s } v \left(2 r_{u}^{2}+(r \pm d) r_{v} \sin v \cos v\right.\right. \\
& \left.+(r \pm d)^{2} \cos ^{2} v-(r \pm d) r_{u u}\right)\left((r \pm d)^{2}+r_{v}^{2}\right) \\
& +\cos v\left(2 r_{v}^{2}+(r \pm d)^{2}-(r \pm d) r_{v v}\right)\left((r \pm d)^{2} \cos ^{2} v+r_{u}^{2}\right) \\
& \left.+2 r_{u} r_{v}\left((r \pm d) r_{u v} \cos v-2 r_{u} r_{v} \cos v+(r \pm d) r_{u} \sin v\right)\right]
\end{aligned}
$$

where $A=\left((r \pm d)^{2}+r_{v}^{2}\right) \cos ^{2} v+r_{u}^{2}[4]$.

Let $M$ be a surface of revolution generated by the rotating curve $c(t)$. The surface is given with the surface patch

$$
X(t, s)=(r(t) \cos t, r(t) \sin t \cos s, r(t) \sin t \sin s),
$$


where $c(t)=r(t)(\cos t, \sin t)$. The coefficients of the first fundamental form of the surface $M$ hold:

$$
\begin{aligned}
& e=r^{2}+\left(r^{\prime}\right)^{2}, \\
& f=0, \\
& g=r^{2} \sin ^{2} t .
\end{aligned}
$$

The Gaussian and mean curvatures of the surface $M$ are as follows:

$$
K=\frac{\left(r^{\prime} \cos t-r \sin t\right)\left(r r^{\prime \prime}-2\left(r^{\prime}\right)^{2}-r^{2}\right)}{\left.r \sin t\left(r^{2}+\left(r^{\prime}\right)^{2}\right)\right)^{3}},
$$

and

$$
H=\frac{r \sin t\left(r r^{\prime \prime}-2\left(r^{\prime}\right)^{2}-r^{2}\right)+\left(r^{2}+\left(r^{\prime}\right)^{2}\right)\left(r^{\prime} \cos t-r \sin t\right)}{2 r \sin t\left(r^{2}+\left(r^{\prime}\right)^{2}\right)^{3 / 2}},
$$

respectively. Let $M_{d}$ be a surface of revolution generated by the conchoid curve $c_{d}(t)$. The surface is parametrized by

$$
\widetilde{X}(t, s)=((r(t) \pm d) \cos t,(r(t) \pm d) \sin t \cos s,(r(t) \pm d) \sin t \sin s),
$$

where $c_{d}(t)=(r(t) \pm d)(\cos t, \sin t)$. The coefficients of the first fundamental form of the surface $M_{d}$ are calculated as

$$
\begin{aligned}
\widetilde{e} & =(r(t) \pm d)^{2}+\left(r^{\prime}\right)^{2}, \\
\widetilde{f} & =0, \\
\widetilde{g} & =(r(t) \pm d)^{2} \sin ^{2} t .
\end{aligned}
$$

The Gaussian and mean curvatures of the surface $M_{d}$ become

$$
\begin{gathered}
\widetilde{K}=\frac{\left(r^{\prime} \cos t-(r(t) \pm d) \sin t\right)\left((r(t) \pm d) r^{\prime \prime}-2\left(r^{\prime}\right)^{2}-(r(t) \pm d)^{2}\right)}{\left.(r(t) \pm d) \sin t\left((r(t) \pm d)^{2}+\left(r^{\prime}\right)^{2}\right)\right)^{3}} \\
\widetilde{H}=\frac{(r(t) \pm d) \sin t\left((r(t) \pm d) r^{\prime \prime}-2\left(r^{\prime}\right)^{2}-(r(t) \pm d)^{2}\right)}{2(r(t) \pm d) \sin t\left((r(t) \pm d)^{2}+\left(r^{\prime}\right)^{2}\right)^{3 / 2}} \\
+\frac{\left((r(t) \pm d)^{2}+\left(r^{\prime}\right)^{2}\right)\left(r^{\prime} \cos t-(r(t) \pm d) \sin t\right)}{2(r(t) \pm d) \sin t\left((r(t) \pm d)^{2}+\left(r^{\prime}\right)^{2}\right)^{3 / 2}}
\end{gathered}
$$

respectively [4].

\section{Discussion and Conclusion}

\subsection{An examination of the condition of the conchoidal surface to be a Bonnet surface in $\mathbf{E}^{3}$}

In this section, we will examine condition which is the conchoidal surface to be a Bonnet surface in Euclidean 3-space. Especially, we will deal with the conchoidal surfaces admitting an infinite number of isometries. Thus, it will be sufficient to determine: (a) the conchoidal surfaces of the constant mean curvature and (b) the isothermic Weingarten conchoidal surfaces. 


\section{(a) The conchoidal surfaces of the constant mean curvature}

Let $M$ be a regular surface given with the parametrization (2.7). It is possible that the mean curvature $H$ given by (2.11) is equal to a non-zero constant when the radius function $r(u, v)$ is a constant. This means that the surface $M$ is a sphere.

Example 3.1. Let the radius function be a constant. For $r(u, v)=3$ and $d=1$, the conchoidal surface $M_{d}$ is given by the parametrization

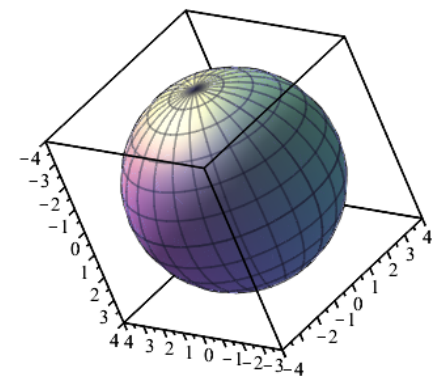

Figure 3.1: Conchoidal surface with $r(u, v)=3$ and $d=1$

$$
X_{d}(u, v)=(4 \cos u \cos v, 4 \sin u \cos v, 4 \sin v) .
$$

It denotes a sphere as given in Figure 3.1.

The mean curvature is a constant when the surface $M$ is minimal, except that the radius function is a constant. In this case, considering [4], if $u$-parameter radius function is

$$
r(u)= \pm \frac{\sqrt{\cos v}}{\sqrt{c_{1} \sin (2 u \cos v)-c_{2} \cos (2 u \cos v)}}
$$

or if $v$-parameter radius function is

$$
r(v)=\frac{1}{c_{1} \sin v},
$$

where $c_{1}, c_{2}$ are constants, then $M$ is the minimal surface. So, the surfaces $M$ determined by (3.2) and (3.3) are the surfaces of the type $(i)$ which can be recognised by an infinite number of isometries preserving the principal curvature.

Similar results for conchoidal surface $M_{d}$ are obtained as follows:

If the radius function is a constant, the mean curvature $\widetilde{H}$ of the conchoidal surface is equal to $\frac{1}{r \pm d}$. This means that the surface $M_{d}$ is a sphere. If $u$-parameter 
radius function is

$$
r(u)= \pm \frac{\sqrt{\cos v}}{\sqrt{c_{1} \sin (2 u \cos v)-c_{2} \cos (2 u \cos v)}} \pm d
$$

or if $v$-parameter radius function is

$$
r(v)=\mp d+\frac{1}{c_{1} \sin v},
$$

where $c_{1}, c_{2}$ are constants, then the surface $M_{d}$ is minimal. So, the conchoidal surfaces $M_{d}$ determined by (3.4) and (3.5) are the conchoidal surfaces of the type (i) which can be recognised by an infinite number of isometries preserving the principal curvature.

Example 3.2. Let the radius function is given by

$$
r(u)=\frac{\sqrt{\cos v}}{\sqrt{\sin (2 u \cos v)-\cos (2 u \cos v)}}
$$

and $d=-1$. Then, the conchoidal surface $M_{d}$ is parametrized by

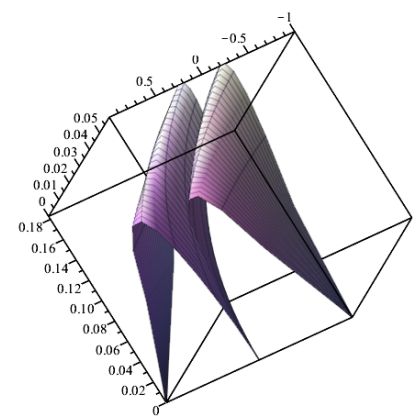

Figure 3.2: Conchoidal surface with $r(u)$ and $d=-1$

$$
X_{d}(u, v)=(r(u)-1)(\cos u \cos v, \sin u \cos v, \sin v) .
$$

It is shown as given in Figure 3.2.

Example 3.3. Let the radius function is given by $r(v)=\frac{1}{2 \sin v}$ and $d=-1$. Then, the conchoidal surface $M_{d}$ is parametrized by

$$
X_{d}(u, v)=\left(\frac{1}{2 \sin v}-1\right)(\cos u \cos v, \sin u \cos v, \sin v) .
$$

It is shown as given in Figure 3.3. 


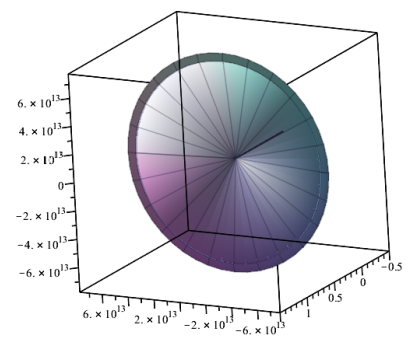

Figure 3.3: Conchoidal surface with $r(v)$ and $d=-1$

(b) The isothermic Weingarten conchoidal surfaces of the non-constant mean curvature

Firstly, let's calculate the condition which is satified by the surface $M$ to be an isothermal surface. When the curves of an orthogonal system have the constant geodesic curvature, the system is an isothermal [6]. For this, we assume that the parameter curves of the surface $M$ constitute the orthogonal system, namely, $\left\langle X_{u}, X_{v}\right\rangle=0$. When the surface is assigned by these parametric curves and the linear element is written $d s^{2}=e d u^{2}+g d v^{2}$, from [6], the condition that the geodesic curvature is a constant becomes $\frac{\partial^{2}}{\partial u \partial v}\left(\log \frac{g}{e}\right)=0$.

When the parameter curves are orthogonal, $\left\langle X_{u}, X_{v}\right\rangle=r_{u} r_{v}=0$. This means that $r_{u}=0$ or $r_{v}=0$. Therefore the parametric curves of the conchoidal surface $M_{d}$ are orthogonal. Thus, when the surface $M$ is isothermal, the obtained cases are valid for the conchoidal surface $M_{d}$. So, we have the following cases:

Case 1: We assume that $r_{u}=0$ and $r_{v} \neq 0$. In order to examine whether the surface $M$ with the radius function $r\left(u_{0}, v\right)$ is a Bonnet surface, we will work the isothermic Weingarten surfaces.

Using (2.9) into (2.6), then we obtain as follows:

$$
\frac{\partial^{2}}{\partial u \partial v}\left(\log \frac{r^{2}+r_{v}^{2}}{r^{2} \cos ^{2} v}\right)=0 .
$$

From (3.9), we conclude that the surface $M$ with the radius function $r\left(u_{0}, v\right)$ is the isothermal surface.

Secondly, we investigate the necessary conditions for the surface $M$ to be a Weingarten surface. Differentiating (2.10) and (2.11) with respect to $u$ and considering $r_{u}=0$, then we find $\frac{\partial K}{\partial u}=0$ and $\frac{\partial H}{\partial u}=0$. Hence, the surface $M$ with the radius function $r\left(u_{0}, v\right)$ is the Weingarten surface. Additionally, from (2.11), we see that the mean curvature of the surface $M$ with the radius function $r\left(u_{0}, v\right)$ is the non-constant. 
As a result, since the surface $M$ is both the isothermal and Weingarten surface with the non-constant mean curvature, then it has an infinite number of the Bonnet nets. Thus, the following theorem is given.

Theorem 3.1. The surface $M$ with the radius function $r\left(u_{0}, v\right)$ is a surface of the type (ii) which admits an infinite number of isometries. So, this surface is a Bonnet surface.

Let $M_{d}$ be a conchoidal surface of $M$ given with the parametrization ( 2.8). If the radius function $r(u, v)$ is a $v$-parameter function, then the coefficients of the first fundamental form of the surface $M_{d}$ are

$$
\begin{aligned}
\widetilde{e} & =(r \pm d)^{2} \cos ^{2} v \\
\widetilde{f} & =0 \\
\widetilde{g} & =(r \pm d)^{2}+r_{v}^{2} .
\end{aligned}
$$

Considering these coefficients, the conchoidal surface $M_{d}$ of $M$ with the radius function $r\left(u_{0}, v\right)$ is the isothermic surface, since we get

$$
\frac{\partial^{2}}{\partial u \partial v}\left(\log \frac{(r \pm d)^{2}+r_{v}^{2}}{(r \pm d)^{2} \cos ^{2} v}\right)=0
$$

To determine the necessary condition to be a Weingarten surface of $M_{d}$, we have (2.12) and (2.13) for $r_{u}=0$. From $\frac{\partial \widetilde{K}}{\partial u}=0$ and $\frac{\partial \widetilde{H}}{\partial u}=0$, the conchoidal surface $M_{d}$ of $M$ with the radius function $r\left(u_{0}, v\right)$ is the Weingarten surface. From (2.13), it is easily seen that $\widetilde{H} \neq$ const. Therefore, the following theorem is given for the conchoidal surface $M_{d}$.

Theorem 3.2. The conchoidal surface $M_{d}$ with the radius function $r\left(u_{0}, v\right)$ is a surface of the type (ii) which admits an infinite number of isometries. So, this surface is a Bonnet surface.

Corollary 3.1. There is no surfaces $M$ and $M_{d}$ that admits a single non-trivial isometry with the non-constant mean curvature.

Example 3.4. Let the radius function is given by $r(v)=\frac{1}{\cos v}$ and $d=2$. Then, the conchoidal surface $M_{d}$ is parametrized by

$$
X_{d}(u, v)=\left(\frac{1}{\cos v}+2\right)(\cos u \cos v, \sin u \cos v, \sin v) .
$$

It is a Bonnet surface and shown as given in Figure 3.4. 


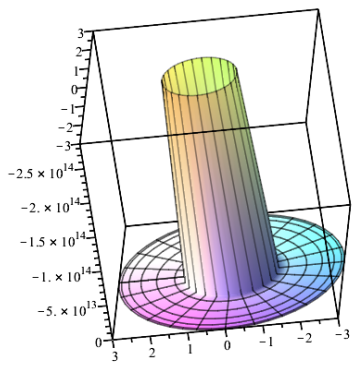

Figure 3.4: Conchoidal surface with $r(v)=\frac{1}{\cos v}$ and $d=2$

Case 2: We assume that $r_{v}=0$ and $r_{u} \neq 0$. In order to examine whether the surface $M$ with the radius function $r\left(u, v_{0}\right)$ is a Bonnet surface, we will study this kind of surface to be the isothermic Weingarten surface.

Using (2.9) into (2.6), then we obtain as follows:

$$
\frac{\partial^{2}}{\partial u \partial v}\left(\log \frac{r^{2}}{r^{2} \cos ^{2} v+r_{u}^{2}}\right)=\frac{2 r r_{u} \sin 2 v\left(r_{u}^{2}-r r_{u u}\right)}{\left(r^{2} \cos ^{2} v+r_{u}^{2}\right)^{2}} .
$$

For $\frac{\partial^{2}}{\partial u \partial v}\left(\log \frac{g}{e}\right)=0$, there exists $r_{u}^{2}-r r_{u u}=0$ from (3.13), that is, the surface $M$ admitting $r_{u}^{2}-r r_{u u}=0$ is an isothermic surface. When we solve this differential equation, we find $r(u)=e^{c_{1} u} c_{2}$, where $c_{1}, c_{2}$ are constants. Thus, the following theorem can be written.

Theorem 3.3. The surface $M$ with the radius function $r\left(u, v_{0}\right)$ is an isothermic surface if and only if it is parametrized by

$$
X(u, v)=e^{c_{1} u} c_{2}(\cos u \cos v, \sin u \cos v, \sin v) .
$$

Let $M_{d}$ be a conchoidal surface of $M$ given with the parametrization ( 2.8). If the radius function $r(u, v)$ is a $u$-parameter function, then the coefficients of the first fundamental form of the surface $M_{d}$ are

$$
\begin{aligned}
\widetilde{e} & =(r \pm d)^{2} \cos ^{2} v+r_{u}^{2}, \\
\widetilde{f} & =0, \\
\widetilde{g} & =(r \pm d)^{2} .
\end{aligned}
$$

Considering these coefficients for the conchoidal surface $M_{d}$ of $M$ with the radius function $r\left(u, v_{0}\right)$, we get

$$
\frac{\partial^{2}}{\partial u \partial v}\left(\log \frac{(r \pm d)^{2}}{(r \pm d)^{2} \cos ^{2} v+r_{u}^{2}}\right)=\frac{2(r \pm d) r_{u} \sin 2 v\left(r_{u}^{2}-(r \pm d) r_{u u}\right)}{\left((r \pm d)^{2} \cos ^{2} v+r_{u}^{2}\right)^{2}} .
$$


For $\frac{\partial^{2}}{\partial u \partial v}\left(\log \frac{g}{e}\right)=0$, there exists $r_{u}^{2}-(r \pm d) r_{u u}=0$ from (3.16), that is, the surface $M$ admitting $r_{u}^{2}-(r \pm d) r_{u u}=0$ is an isothermic surface. Solving this differential equation, then we obtain $r(u)=e^{c_{1} u} c_{2} \mp d$, where $c_{1}, c_{2}$ are constants. Thus, the following theorem can be written.

Theorem 3.4. The conchoidal surface $M_{d}$ with the radius function $r\left(u, v_{0}\right)$ is an isothermic surface if and only if it is parametrized by

$$
X_{d}(u, v)=\left(e^{c_{1} u} c_{2} \mp d\right)(\cos u \cos v, \sin u \cos v, \sin v) .
$$

Secondly, we investigate the necessary condition for the surface $M$ to be a Weingarten surface, namely $\frac{\partial K}{\partial u} \frac{\partial H}{\partial v}-\frac{\partial K}{\partial v} \frac{\partial H}{\partial u}=0$. Differentiating (2.10), (2.11) and considering $r_{v}=0$, then we get

$$
\frac{\partial K}{\partial u} \frac{\partial H}{\partial v}-\frac{\partial K}{\partial v} \frac{\partial H}{\partial u}=\frac{2 c_{1}^{3} \sin v\left(-\cos ^{4} v+2 \cos ^{2} v+c_{1}^{2}\right)}{c_{2}^{3} e^{3 c_{1} u}\left(\cos ^{2} v+c_{1}^{2}\right)^{7 / 2}}
$$

If $(3.18)$ is equal to zero, then $\left(\cos ^{2} v-1\right)^{2}=c_{1}^{2}+1$. Thus, $\cos v$ is a constant and this contradicts with $M$, which is defined (3.14), being a surface. There is no surface $M$ given by (3.14) that is a Weingarten surface and so, the surface $M$ with the radius function $r\left(u, v_{0}\right)$ is not a Bonnet surface. When we examine the conchoidal surface $M_{d}$, we get similar results. There is no surface $M_{d}$ given by (3.17) that is a Weingarten surface and so, the surface $M_{d}$ with the radius function $r\left(u, v_{0}\right)$ is not a Bonnet surface.

Example 3.5. Let the radius function is given by $r(u)=2 e^{u}$ and $d=1$. Then, the conchoidal surface $M_{d}$ is parametrized by

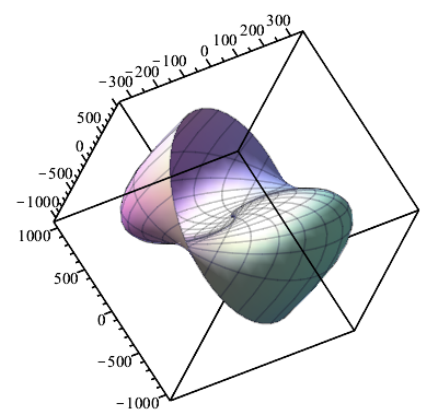

Figure 3.5: Conchoidal surface with $r(u)=2 e^{u}$ and $d=1$

$$
X_{d}(u, v)=\left(2 e^{u}+1\right)(\cos u \cos v, \sin u \cos v, \sin v)
$$

It is the isothermic surface, however it is not the Weingarten surface. Thus, it is not a Bonnet surface and it is shown as given in Figure 3.5. 


\subsection{An examination of the condition of the surface of revolution given with conchoid curve to be a Bonnet surface in $\mathbb{E}^{3}$}

In this section, we will examine condition which is the surface of revolution given with the rotating curve $c(t)$ and its the conchoid curve $c_{d}(t)$ to be a Bonnet surface.

\section{(a) The surfaces of revolution of the constant mean curvature}

Assume that $M$ and $M_{d}$ are the surfaces of revolution generated by the rotating curve $c(t)$ and its conchoid curve $c_{d}(t)$ parametrized by (2.14) and (2.18). It is possible that the mean curvature $H$ given by (2.17) is equal to a non-zero constant when the radius function $r(t)$ is a constant. This means that the surfaces $M$ and $M_{d}$ are the spheres.

Example 3.6. Let $M_{d}$ be a surface of revolution generated by the conchoid curve $c_{d}(t)=$ 5. Then, its parametrization is given by

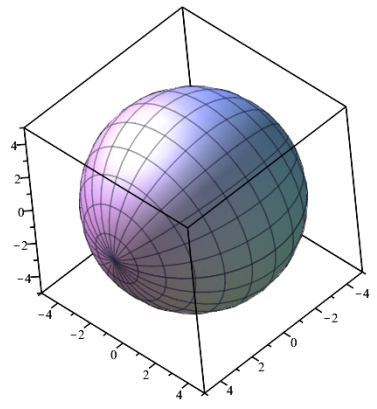

Figure 3.6: Surface of revolution with a constant radius function

$$
X_{d}(t, s)=(5 \cos t, 5 \sin t \cos s, 5 \sin t \sin s) .
$$

It denotes a sphere and it is shown as given in Figure 3.6.

Their mean curvatures are constants when the surfaces $M$ and $M_{d}$ are the minimal surfaces. According to that, considering [4], if the radius function is $r(t)=\frac{c}{\cos t}$, the surface $M$ is a minimal and if the radius function is $r(t)= \pm d+\frac{c}{\cos t}$, the surface $M_{d}$ is a minimal. So, the surfaces $M$ and $M_{d}$ are the surfaces of the type $(i)$ which can be recognised by an infinite of isometries preserving the principal curvatures where $M$ is determined by ( 2.14) with $r(t)=\frac{c}{\cos t}$ and $M_{d}$ is determined by (2.18) with $r(t)= \pm d+\frac{c}{\cos t}$. 


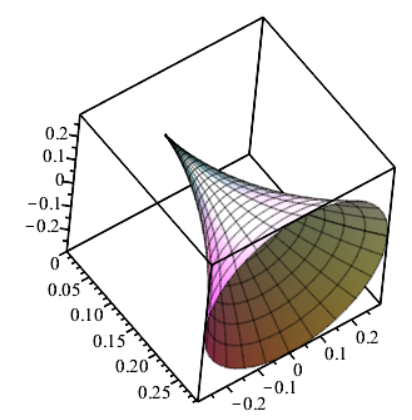

Figure 3.7: Surface of revolution with $c_{d}(t)=\left(\frac{1}{\cos t}-1\right)(\cos t, \sin t)$

Example 3.7. Let $M_{d}$ be a surface of revolution generated by the conchoid curve $c_{d}(t)=$ $\left(\frac{1}{\cos t}-1\right)(\cos t, \sin t)$. Then, its parametrization is given by

$$
X_{d}(t, s)=\left(\frac{1}{\cos t}-1\right)(\cos t, \sin t \cos s, \sin t \sin s)
$$

It is shown as given in Figure 3.7.

(b) The isothermic Weingarten surface of revolution of the non-constant mean curvature

According to (2.15), from $f=0$, we see that the parameter curves of the surface $M$ constitute the orthogonal system. Similarly, from $\widetilde{f}=0$, the parameter curves of the surface of revolution $M_{d}$ are the orthogonal system.

Firstly, we consider the surface providing the condition $\frac{\partial^{2}}{\partial t \partial s}\left(\log \frac{g}{e}\right)=0$ since every Bonnet surface is an isothermic surface. For the surface $M$, using (2.15), then we have $\frac{\partial^{2}}{\partial t \partial s}\left(\log \frac{r^{2} \sin ^{2} t}{r^{2}+\left(r^{\prime}\right)^{2}}\right)=0$.

Then, we need to show the necessary condition for the surface of revolution $M$ to be a Weingarten surface. From $(2.5),(2.16)$ and $(2.17)$, we find $\frac{\partial K}{\partial s}=0$ and $\frac{\partial H}{\partial s}=0$. So, the surface of revolution $M$ is the isothermic Weingarten surface.

Using (2.17), we realize that the mean curvature of the surface $M$ is a nonconstant. Hence, the surface of revolution $M$ generated by the rotating curve $c(t)$ with the non-constant mean curvature is the Bonnet surface since it is the isothermic Weingarten surface. Also, if we study the surface of revolution $M_{d}$ generated by the conchoid curve $c_{d}(t)$ with the help of the above calculations, then we conclude that the surface $M_{d}$ is the Bonnet surface. 
Theorem 3.5. The surface of revolution $M$ parametrized by (2.14) and the surface of revolution $M_{d}$ parametrized by (2.18) are the surfaces of the type (ii) which admit an infinite number of isometries. So, the surfaces of revolution $M$ and $M_{d}$ are the Bonnet surfaces.

Corollary 3.2. There is no surface of revolution given with the conchoid curve that permits a single non-trivial isometry with the non-constant mean curvature.

Example 3.8. Let $M_{d}$ be a surface of revolution generated by the conchoid curve $c_{d}(t)=$ $(2 \sin t+2)(\cos t, \sin t)$. Then, its parametrization is given by

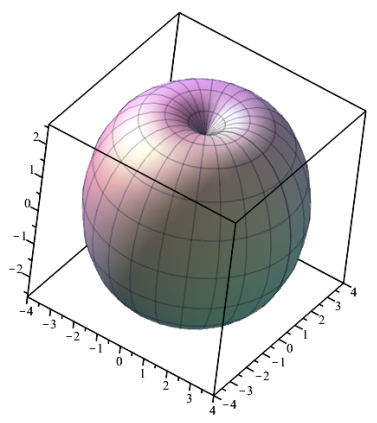

Figure 3.8: Surface of revolution with $c_{d}(t)=(2 \sin t+2)(\cos t, \sin t)$

$$
X_{d}(t, s)=(2 \sin t+2)(\cos t, \sin t \cos s, \sin t \sin s) .
$$

It is shown as given in Figure 3.8 and it is a Bonnet surface.

\section{References}

1. G. Aydin Şekerci and M. Çımdiker: Bonnet canal surfaces. Dokuz Eylul University Fac. of Engineering J. of Sci. and Engineering 21 (2019), 195-200.

2. R. M. A. Azzam: Limacon of Pascal locus of the complex refractive indices of interfaces with maximally flat reflectance-versus-angle curves for incident unpolarized light. J. Opt. Soc. Am. Opt. Imagen Sci. Vis. 9 (1992), 957-963.

3. O. Bonnet: Memoire sur la theorie des surfaces applicables sur une surface donnee. J. Ec. Polytech. Math. (1867), 72-92.

4. B. Bulca, S. N. OruÇ and K. Arslan: Conchoid curves and surfaces in Euclidean 3-space. BAUN Inst. Sci. Technol. 20(2) (2018), 467-481.

5. P. M. do Carmo: Differential Geometry of Curves and Surfaces. Prentice-Hall, USA, 1976. 
6. L. P. Eisenhart: A Treatise On the Differential Geometry of Curves and Surfaces. Dover Publications, Inc., Newyork, 1960.

7. S. ERsoy and K. EREN: Timelike tangent developable surfaces and Bonnet surfaces. Abstract and Applied Analysis 2016 (2016), 1-7.

8. A. GRAY: Modern Differential Geometry of Curves and Surfaces with Mathematica. CCR Press, New York, 1997.

9. A. H. KerRICK: The limacon of Pascal as a basis for computed and graphic methods of determining astronomic positions. J. Inst. Navigat. 6(5) (1959), 310316.

10. F. Kanbay: Bonnet ruled surfaces. Acta Math. Sin. Engl. Ser. 21 (2005), 623630 .

11. V. Lalan: Les formes minima des surfaces d'ossian Bonnet. Bull. Soc. Math., France 77 (1949), 102-127.

12. E. H. Lockwood: A Book of Curves. Cambridge University Press, London, 1961.

13. B. Odehnal: Generalized conchoids. KoG 21 (2017), 35-46.

14. B. O'NeILL: Elementary Differential Geometry. Academic Press, USA, 1997.

15. M. Peternell, D. Gruber and J. Sendra: Conchoid surfaces of rational ruled surfaces. Computer Aided Geometric Design 28(7) (2011), 427-435.

16. M. Peternell, D. Gruber and J. Sendra: Conchoid surfaces of spheres. Computer Aided Geometric Design. 30(1) (2013), 35-44.

17. M. Peternell, L. Gotthart, J. Sendra and J. R. Sendra: Offsets, conchoids and pedal surfaces. Journal of Geometry 106 (2015), 321-339.

18. Z. SOYUÇOK: The problem of non-trivial isometries of surfaces preserving principal curvatures. J. Geom. 52 (1995), 173-188.

19. F. Szmulowicz: Conchoid of Nicomedes from reflections and refractions in a cone. Amer. J. of Phys. 64 (1996), 467-471. 